\title{
Intelligent Detection of Bad Credit Card Accounts
}

\author{
Yo-Ping Huang1, Frode Eika Sandnes ${ }^{2}$, Tsun-Wei Chang 3 \&Chun-Chieh Lu ${ }^{4}$ \\ ${ }^{1}$ Dept. of Electrical Engineering, National Taipei University of Technology, Taiwan \\ ${ }^{2}$ Faculty of Engineering, Oslo University College, Norway \\ ${ }^{3}$ Dept. of Computer Science and Information Eng., De Lin Institute of Technology, Taiwan \\ ${ }^{4}$ Department of Computer Science and Engineering, Tatung University, Taiwan
}

\section{Overview}

The effective management of credit risk is a critical component of risk management and essential to the long-term success of any banking organization. Credit-risk management practices vary considerably among organizations. Bad accounts affect the credit reputation of the cardholder and cause issuers to loose billions of dollars each year. However, bad debts problems are complex, full of uncertainty and can only be resolved through manual intervention. This study proposes an intelligent model for the early and accurate detection of bad accounts based on fuzzy logic (FL) and back propagation neural networks (BPN). Experimental results verify the effectiveness of the proposed strategy.

\section{Introduction}

The number of credit cards issued in Taiwan has increased rapidly since 1992. In 2004 the number of cards totalled approximately 44,180,000 cards, and the amount of revolving credit had reached NT $\$ 457,900$ million (new Taiwan dollars) (Central Bank of China, 2008). Due to the over-issue credit cards, the amount of bad debt has reached NT\$21,400 million. Cardholders with financial difficulties may be unable to keep up with payments. The card issuing banks subsequently may risk financial losses. According to the 2004 annual report of bad debt amount of revolving credit from Taiwan Financial Supervisory Commission (FSC) (Taiwan Financial Supervisory Commission, 2008) reveals that many banks suffer large and the losses are characterized by a year-on-year growth of $10.2 \%$ (Lin, 2005).

The FSC began to pay attention to the problem to prevent the Taiwanese credit card market from repeating the South Korea financial crisis (The International Commercial Bank of China, 2008). Bad credit card accounts are classified into two types: One type covers bad debt accounts that have bad payment records. It has a potential risk to regain credit amount. The other is called a charge-off account, where customers show no willingness to pay the debt, and the issuer will have to write off the debt as a loss. 
Bad accounts not only result in losses for the issuers but also affect the reputation of cardholders which is maintained by the Taiwan Joint Credit Information Center (JCIC) (Taiwan Joint Credit Information Center, 2008). The JCIC will store information about charge-off accounts for seven years. Card issuers are responsible for reporting bad accounts to the JCIC and once a customer has a bad credit entry in the JCIC it may be more difficult, or impossible, to borrow money.

The process of detecting bad accounts is fraught with difficulties. The problem of bad account detection is complicated, uncertain, and is dependent on the operation environment. Many bad account records are not caught by current detection systems.

The primary objective of this study is to discover important credit card patterns that can be used for detection. This is achieved by soliciting good debt, bad debt, and charge-off records from a credit card company database. An intelligent fuzzy-neural inference system (Chakraborty, 2001; Huang et. al., 2007) is utilized to construct a better prediction model.

\section{Background}

A credit card allows a cardholder with a credit limit to purchase goods and services to obtain cash disbursements on credit offered by a third party, for which a cardholder is subsequently billed (National Credit Card Center of R.O.C, 2008). There are six parties involved in a credit card transaction (ShopSite, 2008), namely cardholders, issuers, merchants, acquirers, i.e., a bank or other financial institution that approves a merchant for accepting credit cards, and then collects the merchant's online payments, associations, e.g., Visa and MasterCard are associations of member banks and financial institutions (American Express, Discover, and Diner's Club are single corporations that issue their own cards), payment gateways, i.e., a company that provides an interface between the Internet and the secure banking networks. A payment gateway authenticates the parties involved and acts as a channel for moving credit card transactions from a store to a payment processor.

First, the customer applies for a credit card from the issuer (Cardservice International, 2008; Indiana University Office of the Treasurer, 2008). A bank or other financial institution issues the credit card according to the applicant's credit status, income and reimbursement ability. Then, the customer selects the desired products or services and pays a merchant using the card, whereupon the merchant's credit card terminal sends the request to the merchant's payment gateway. The information includes the store's identification information, the customer's credit card information, and the amount. The payment gateway checks its database to see which acquirer the store uses. The gateway sends the transaction information to the acquirer's payment processor. Then, the payment processor examines the customer's credit card number to determine the issuer and forwards the customer information and amount. Next, the issuer checks if the information is valid and if there is enough credit to cover the transaction. If ok, the issuer sends an authorization code or declined transaction message back to the payment processor which again sends the authorization message back to the payment gateway. Next the store issues a receipt to the customer and the issuing banks settle funds to the acquiring bank. The acquirer deposits the 
money in the merchant's local account, minus the discount rate and transaction fee. The issuer's billing system computes cardholder's transactions, fee, and interest on cycle day and sends a bill to notify cardholder to pay within the due-date.

\subsection{Credit Card Risk Management}

Some banks are improving the credit decision-making process by means of credit scoring technologies that often are based on expert systems that can adapt to changes in the economy or within specific customer segments. If a customer scores low on the scoring system their application will be rejected or accepted with only a low credit line. The use of credit-scoring varies from those that are using only credit bureau data and JCIC data, to those that blend bureau data with other information based on the firm's own experience, to the most advanced applications using adaptive algorithms (Burns \& Stanley, 2001).

Faced with an exploding number of transactions, experienced personnel are no longer able to identify fraud caused by stolen cards, false merchants or disloyal employees. Automatic fraud detection has therefore become an important topic (Kuo et. al., 2004).

\subsection{Data Mining}

Data mining aims to discover hidden knowledge, unknown patterns, and new rules from large databases that are potentially useful and ultimately understandable for making crucial decisions (Zhang \& Zhou, 2004).

The knowledge to be mined is closely related to a target application and the original data. Therefore, data mining should be considered along with several other issues rather than an isolated task. First, data mining needs to take ultimate applications into account. For example, credit card fraud detection and stock market prediction may require different data mining techniques.

\subsection{Credit Card Data Mining}

In recent years data mining approaches (Zhang \& Zhou, 2004) have been proposed for solving credit card related problems. For instance, Yu (2003) addressed the detection of bad debt and inactive accounts from the customer profile and delinquency. Chiang (2003) addressed the relationship between credit-evaluation factors and credit card default risk. Lee (2002) analysed the credit risk of cardholders based on their profile. Lin (2003) addressed the risk improvement of cardholders based on their payment record. Aleskerov et. al. (1997) and Brause et. al. (1999) both addressed credit card fraud detection using transaction category, amount and time.

\subsection{Fuzzy Logic}

Fuzzy logic systems (FLS) usually contain four major components (Huang et. al., 2006): Fuzzification, i.e., the relationships between input variables and linguistic variables is defined by using membership functions (MFs). Knowledge Base, i.e., application domain expert knowledge. It uses the linguistic rules to represent an expert's tactic. Decision Making Logic, i.e., the inference engine has the capacity of inferring rules using fuzzy rules and fuzzy implication, and so simulates the human decision-making ability. Defuzzification, i.e., 
the reverse process of fuzzificaiton. It produces a crisp output value from the fuzzy outputs of the inference engine.

\subsection{Back-Propagation Neural Network}

Neural networks (NNs) have been widely applied to various tasks in hybrid combinations with data mining approaches (Huang et. al., 2008; Huang et. al., 2007; Kijsirikul \& Chongkasemwongse, 2001). NNs are computer models built to mimic human pattern recognition. NNs are able to learn the unknown non-linear relationships between causes and prospective outcomes based on examples.

\subsection{BPN Model}

The back-propagation neural network (BPN) is one of the most popular artificial supervised learning neural networks. It is very powerful in terms of assessment and prediction. A neural network consists of a set of fundamental processing elements (also called neurons) that are distributed in hierarchical layers. Most multi-layer feed-forward neural networks contain three types of layers, namely the input layer, the hidden layer and the output layer. The input layer receives features of input data and distributes them to the hidden layer. After each neuron in a hidden layer receives the inputs from all of the neurons in a layer ahead of input layer, the values are added through applied weights and converted to an output value by an activation function. Then, the output is passed to all of the neurons in the next layer, providing a feed-forward path to the output layer. The weights between two neurons in two adjacent layers are adjusted through an iterative training process while training samples are presented to the network. The output layer yields the outputs of the network.

\subsection{BPN Algorithm}

The BPN algorithm applies the basic principle of the gradient steepest descent method to minimize the error function. It compares the outputs of the processing units in the output layer with desired outputs to adjust the connecting weights. The weights between two neurons in two adjacent layers are adjusted through an iterative training process while training samples are presented to the network. A close approximation of the transformation function which is compared the outputs of the processing units in the output layer with desired outputs can be acquired (Huang \& Hsieh, 2003). After training, the network can be used to classify unseen data. The unseen data are then fed into the network, and the output with highest value will be taken as the prediction.

\section{Method}

\subsection{Training and test material}

Experimental data were collected randomly from a local bank in Taiwan on June 30, 2004. In order to reduce noise and handle missing values, the stop card accounts and unreasonable accounts were discarded from the dataset. The original dataset consisted of 744,477 accounts which were reduced to 449,211 records after a cleanup. The validation samples comprised 446,211 accounts including 432,916 (97.02\%) normal accounts, 9,105 (2.04\%) bad accounts and 4,190 (0.94\%) charge-off accounts. The data set was divided to a training sample and a test sample. The training samples consisted of 3,000 accounts upon which 1,000 (33.33\%) 
were bad accounts, 1,000 (33.33\%) were charge-off accounts, and 1,000 (33.33\%) were normal accounts.

Input variables that deal with cardholder's accounts were divided into two groups: (1) socio-economic data and (2) financial data. Basic socio-economic characteristics that are used in our raw database are: (1) gender, (2) marital status, (3) education, (4) age and (5) occupation. Basic financial characteristics that are used in our raw database are: (1) credit limit, (2) current balance, (3) payment amount, (4) transaction amount, (5) revolving credit amount, (6) late charge fee, (7) credit cash amount, (8) delinquency flag, (9) cycle, (10) client's account age and (11) zip code.

Clients' accounts are classified as being either normal, bad debt, or charge-off. Clients are in bad debt if they exceed a contracted overdraft for more than 30 days during a period of 6 months. Clients are charge-off if they exceed a contracted overdraft for more than 180 days during a period of 6 months. Otherwise, a client is considered normal.

\subsection{Data Selection}

The scheme employed herein incorporates the following features: (1) Credit limit, the maximum amount a person is allowed to borrow on a credit card (see Table 1). It includes purchases, cash advances, and any finance charges or fees. Some issuers increase cardholder's credit limit to promote their consumption. Most of the bad account's credit limit is below NT $\$ 100,000$. The normal account's credit limit is between NT\$100,000 and NT\$300,000. (2) Gender, Table 2 shows the credit status related to gender. Female clients have a higher rate of normal accounts than male clients. Males therefore have higher risk of bad accounts than females. (3) Education, Table 3 shows the cardholder's education. Clients with good education have higher rate of normal accounts than other groups. Accounts with just a high-school diploma are at higher risk than others. (4) Marital status, the data revealed that the credit status has no apparent relationship to marital status. (5) Cycle, a monthly billing date from a creditor which summarizes the activity and expenses on an account between the last billing date and the current billing date. The effect of cycle on credit status shows no apparent difference in the entire classes as given in Table 3. (6) Age, there is a group of high-risk cardholders between 20-40 years of age as shown in Table 4 . Note that workers younger than 20 years old or elder than 65 years old who are unemployed are discarded (see Table 4). (7) Client account age, clients who have had accounts for about one year make up a group of high-risk cardholders. Normal account holders continue using their credit cards without problems beyond the first year as shown in Table 5. (8) Current balance, the total amount of money owed on a credit line. It includes any unpaid balance from the previous months, new purchases, cash advances and any charges at present. There are $91.78 \%$ normal accounts owed below NT\$100,000 dollars as given in Table 6. (9) Payment amount paid before the next billing date, the bad accounts and charge-off accounts have low payment amounts. They have no ability to pay off their credit amount as shown in Table 6 . (10) Transaction amount, the amount that a person charges and owes on a credit card between the last billing date and the current billing date. It includes purchases, cash advances, and any finance charges or fees. The account of poor credit status will be limited their purchase as shown in Table 7. (11) Delinquent flag, a credit line or loan account where the late payments have been received or the payments have not been made according to the 
respective terms and conditions in a current month. The charge-off account has the current delinquent flag of long term as demonstrated in Table 8. (12) Balance to credit line ratio $(B / C)$, is used to record the cardholder usage of the credit line. The normal accounts use the credit card in a good manner. The charge-off accounts have a high $\mathrm{B} / \mathrm{C}$ ratio with over purchase as shown in Table 9.

\begin{tabular}{|c|c|c|c|c|c|c|c|c|}
\hline \multirow[b]{2}{*}{ Credit limit } & \multicolumn{2}{|c|}{ Normal account } & \multicolumn{2}{|c|}{ Bad debt account } & \multicolumn{2}{|c|}{ Charge-off account } & \multicolumn{2}{|c|}{ Total account } \\
\hline & No. & $\%$ & No. & $\%$ & No. & $\%$ & No. & $\%$ \\
\hline $1-100000$ & 66,090 & $15.2 \%$ & 6,217 & $61.5 \%$ & 3,176 & $61.2 \%$ & 75,483 & $16.8 \%$ \\
\hline $100,001-200,000$ & 117,227 & $27.0 \%$ & 2,179 & $21.6 \%$ & 1,186 & $22.9 \%$ & 120,592 & $26.9 \%$ \\
\hline $200,001-300,000$ & 135,965 & $31.3 \%$ & 1,230 & $12.2 \%$ & 682 & $13.1 \%$ & 137,877 & $30.7 \%$ \\
\hline $300,001-400,000$ & 70,572 & $16.3 \%$ & 328 & $3.3 \%$ & 119 & $2.3 \%$ & 71,019 & $15.8 \%$ \\
\hline $400,001-500,000$ & 26,495 & $6.1 \%$ & 124 & $1.2 \%$ & 20 & $0.4 \%$ & 26,639 & $5.9 \%$ \\
\hline 500,001 and over & 17,567 & $4.1 \%$ & 27 & $0.3 \%$ & 7 & $0.1 \%$ & 17,601 & $3.9 \%$ \\
\hline Total & 433,916 & $100.0 \%$ & 10,105 & $100.0 \%$ & 5,190 & $100.0 \%$ & 449,211 & $100.0 \%$ \\
\hline
\end{tabular}

Table 1. Risk related to credit limit.

\begin{tabular}{|c|c|c|c|c|c|c|c|c|}
\hline \multirow[b]{2}{*}{ Gender } & \multicolumn{2}{|c|}{ Normal account } & \multicolumn{2}{|c|}{ Bad debt account } & \multicolumn{2}{|c|}{ Charge-off account } & \multicolumn{2}{|c|}{ Total account } \\
\hline & No. & $\%$ & No. & $\%$ & No. & $\%$ & No. & $\%$ \\
\hline Female & 291,118 & $67.1 \%$ & 4,841 & $47.9 \%$ & 2259 & $43.5 \%$ & 298,218 & $66.4 \%$ \\
\hline Male & 142,798 & $32.9 \%$ & 5,264 & $52.1 \%$ & 2931 & $56.5 \%$ & 150,993 & $33.6 \%$ \\
\hline Total & 433,916 & $100.0 \%$ & 10,105 & $100.0 \%$ & 5190 & $100.0 \%$ & 449,211 & $100.0 \%$ \\
\hline
\end{tabular}

Table 2. Risk related to gender.

\begin{tabular}{|c|c|c|c|c|c|c|c|c|}
\hline \multirow[b]{2}{*}{ Education } & \multicolumn{2}{|c|}{ Normal account } & \multicolumn{2}{|c|}{ Bad debt account } & \multicolumn{2}{|c|}{ Charge-off account } & \multicolumn{2}{|c|}{ Total account } \\
\hline & No. & $\%$ & No. & $\%$ & No. & $\%$ & No. & $\%$ \\
\hline Master & 19,725 & $4.6 \%$ & 135 & $1.3 \%$ & 27 & $0.5 \%$ & 19,887 & $4.4 \%$ \\
\hline College & 193,883 & $44.7 \%$ & 2,250 & $22.3 \%$ & 843 & $16.2 \%$ & 196,976 & $43.9 \%$ \\
\hline High school & 143,807 & $33.1 \%$ & 5,302 & $52.5 \%$ & 2,953 & $56.9 \%$ & 152,062 & $33.9 \%$ \\
\hline Unknown & 76,501 & $17.6 \%$ & 2,418 & $23.9 \%$ & 1,367 & $26.3 \%$ & 80,286 & $17.9 \%$ \\
\hline Total & 433,916 & $100.0 \%$ & 10,105 & $100.0 \%$ & 5,190 & $100.0 \%$ & 449,211 & $100.0 \%$ \\
\hline
\end{tabular}

Table 3. Risk related to education.

\begin{tabular}{|c|c|c|c|c|c|c|c|c|}
\hline \multirow[b]{2}{*}{ Age } & \multicolumn{2}{|c|}{ Normal account } & \multicolumn{2}{|c|}{ Bad debt account } & \multicolumn{2}{|c|}{ Charge-off account } & \multicolumn{2}{|c|}{ Total account } \\
\hline & No. & $\%$ & No. & $\%$ & No. & $\%$ & No. & $\%$ \\
\hline $20-30$ & 86,977 & $20.0 \%$ & 3,071 & $30.4 \%$ & 1,267 & $24.4 \%$ & 91,315 & $20.3 \%$ \\
\hline $31-40$ & 156,391 & $36.0 \%$ & 2,976 & $29.5 \%$ & 1,617 & $31.2 \%$ & 160,984 & $35.8 \%$ \\
\hline $41-50$ & 119,563 & $27.6 \%$ & 2,550 & $25.2 \%$ & 1,506 & $29.0 \%$ & 123,619 & $27.5 \%$ \\
\hline $51-60$ & 55,934 & $12.9 \%$ & 1,275 & $12.6 \%$ & 678 & $13.1 \%$ & 57,887 & $12.9 \%$ \\
\hline
\end{tabular}




\begin{tabular}{ccccccccc}
\hline & \multicolumn{2}{c}{ Normal account } & \multicolumn{2}{c}{ Bad debt account } & \multicolumn{2}{c}{ Charge-off account } & \multicolumn{2}{c}{ Total account } \\
Age & No. & $\%$ & No. & $\%$ & No. & $\%$ & No. & $\%$ \\
$61-70$ & 12,302 & $2.8 \%$ & 219 & $2.2 \%$ & 112 & $2.2 \%$ & 12,633 & $2.8 \%$ \\
$71-80$ & 2,713 & $0.6 \%$ & 14 & $0.1 \%$ & 10 & $0.2 \%$ & 2,737 & $0.6 \%$ \\
$81-90$ & 33 & $0.0 \%$ & 0 & $0.0 \%$ & 0 & $0.0 \%$ & 33 & $0.0 \%$ \\
90 and over & 3 & $0.0 \%$ & 0 & $0.0 \%$ & 0 & $0.0 \%$ & 3 & $0.0 \%$ \\
Total & 433,916 & $100.0 \%$ & 10,105 & $100.0 \%$ & 5,190 & $100.0 \%$ & 449,211 & $100.0 \%$ \\
\hline
\end{tabular}

Table 4. Comparison of credit status by age.

\begin{tabular}{|c|c|c|c|c|c|c|c|c|}
\hline \multirow[b]{2}{*}{ Account age } & \multicolumn{2}{|c|}{ Normal account } & \multicolumn{2}{|c|}{ Bad debt account } & \multicolumn{2}{|c|}{ Charge-off account } & \multicolumn{2}{|c|}{ Total account } \\
\hline & No. & $\%$ & No. & $\%$ & No. & $\%$ & No. & $\%$ \\
\hline 1 & 22,971 & $5.3 \%$ & 405 & $4.0 \%$ & 0 & $0.0 \%$ & 23,376 & $5.2 \%$ \\
\hline 2 & 80,839 & $18.6 \%$ & 3,854 & $38.1 \%$ & 1,689 & $32.5 \%$ & 86,382 & $19.2 \%$ \\
\hline 3 & 90,434 & $20.8 \%$ & 2,276 & $22.5 \%$ & 1,405 & $27.1 \%$ & 94,115 & $21.0 \%$ \\
\hline 4 & 186,869 & $43.1 \%$ & 2,946 & $29.2 \%$ & 1,697 & $32.7 \%$ & 191,512 & $42.6 \%$ \\
\hline 5 & 8,368 & $1.9 \%$ & 132 & $1.3 \%$ & 119 & $2.3 \%$ & 8,619 & $1.9 \%$ \\
\hline 6 & 15,056 & $3.5 \%$ & 164 & $1.6 \%$ & 101 & $2.0 \%$ & 15,321 & $3.4 \%$ \\
\hline 7 & 11,729 & $2.7 \%$ & 136 & $1.4 \%$ & 66 & $1.3 \%$ & 11,931 & $2.7 \%$ \\
\hline 8 & 7,501 & $1.7 \%$ & 79 & $0.8 \%$ & 47 & $0.9 \%$ & 7,627 & $1.7 \%$ \\
\hline 9 & 3,317 & $0.8 \%$ & 45 & $0.5 \%$ & 22 & $0.4 \%$ & 3,384 & $0.8 \%$ \\
\hline 10 & 1,916 & $0.4 \%$ & 19 & $0.2 \%$ & 19 & $0.4 \%$ & 1,954 & $0.4 \%$ \\
\hline 11 & 1,824 & $0.4 \%$ & 21 & $0.2 \%$ & 17 & $0.3 \%$ & 1,862 & $0.4 \%$ \\
\hline 12 & 2,960 & $0.7 \%$ & 28 & $0.3 \%$ & 7 & $0.1 \%$ & 2,995 & $0.7 \%$ \\
\hline 13 & 132 & $0.0 \%$ & 0 & $0.0 \%$ & 1 & $0.0 \%$ & 133 & $0.0 \%$ \\
\hline Total & 433,916 & $100.0 \%$ & 10,105 & $100.0 \%$ & 5,190 & $100.0 \%$ & 449,211 & $100.0 \%$ \\
\hline
\end{tabular}

Table 5. Risk related to account age.

\begin{tabular}{|c|c|c|c|c|c|c|c|c|}
\hline \multirow[b]{2}{*}{ Current Balance } & \multicolumn{2}{|c|}{ Normal account } & \multicolumn{2}{|c|}{ Bad debt account } & \multicolumn{2}{|c|}{ Charge-off account } & \multicolumn{2}{|c|}{ Total account } \\
\hline & No. & $\%$ & No. & $\%$ & No. & $\%$ & No. & $\%$ \\
\hline 0 & 178,493 & $41.1 \%$ & 2,406 & $23.8 \%$ & 11 & $0.2 \%$ & 180,910 & $40.3 \%$ \\
\hline $1-100,000$ & 219,727 & $50.6 \%$ & 5,568 & $55.1 \%$ & 3,374 & $65.0 \%$ & 228,669 & $50.9 \%$ \\
\hline $100,001-200,000$ & 22,769 & $5.3 \%$ & 1,151 & $11.4 \%$ & 1,046 & $20.2 \%$ & 24,966 & $5.6 \%$ \\
\hline $\begin{array}{c}200,001- \\
300,000\end{array}$ & 8,684 & $2.0 \%$ & 643 & $6.4 \%$ & 560 & $10.8 \%$ & 9,887 & $2.2 \%$ \\
\hline $\begin{array}{l}300,001- \\
400,000\end{array}$ & 3,005 & $0.7 \%$ & 212 & $2.1 \%$ & 136 & $2.6 \%$ & 3,353 & $0.8 \%$ \\
\hline $\begin{array}{c}400,001- \\
500,000\end{array}$ & 981 & $0.2 \%$ & 70 & $0.7 \%$ & 43 & $0.8 \%$ & 1,094 & $0.2 \%$ \\
\hline $\begin{array}{c}500,001- \\
600,000\end{array}$ & 193 & $0.0 \%$ & 41 & $0.4 \%$ & 9 & $0.8 \%$ & 243 & $0.1 \%$ \\
\hline $\begin{array}{l}600,001- \\
700,000\end{array}$ & 26 & $0.0 \%$ & 5 & $0.1 \%$ & 9 & $0.8 \%$ & 40 & $0.0 \%$ \\
\hline Total & 433,916 & $100.0 \%$ & 10,105 & $100.0 \%$ & 5,190 & $100.0 \%$ & 449,211 & $100.0 \%$ \\
\hline
\end{tabular}

Table 6. Risk related to current balance. 


\begin{tabular}{|c|c|c|c|c|c|c|c|c|}
\hline \multirow[b]{2}{*}{ Payment amount } & \multicolumn{2}{|c|}{ Normal account } & \multicolumn{2}{|c|}{ Bad debt account } & \multicolumn{2}{|c|}{ Charge-off account } & \multicolumn{2}{|c|}{ Total account } \\
\hline & No. & Percentage & No. & Percentage & No. & Percentage & No. & Percentage \\
\hline 0 & 334,814 & $77.2 \%$ & 9,546 & $94.5 \%$ & 5,104 & $98.3 \%$ & 349,464 & $77.8 \%$ \\
\hline $1-100,000$ & 64,254 & $14.8 \%$ & 466 & $4.6 \%$ & 75 & $1.5 \%$ & 64,795 & $14.4 \%$ \\
\hline $100,001-200,000$ & 15,023 & $3.5 \%$ & 37 & $0.4 \%$ & 4 & $0.1 \%$ & 15,064 & $3.4 \%$ \\
\hline $200,001-300,000$ & 6,177 & $1.4 \%$ & 20 & $0.2 \%$ & 4 & $0.1 \%$ & 6,201 & $1.4 \%$ \\
\hline $300,001-400,000$ & 3,270 & $0.8 \%$ & 6 & $0.1 \%$ & 0 & $0.0 \%$ & 3,276 & $0.7 \%$ \\
\hline $400,001-500,000$ & 2,358 & $0.5 \%$ & 2 & $0.0 \%$ & 0 & $0.0 \%$ & 2,360 & $0.5 \%$ \\
\hline $500,001-600,000$ & 1,444 & $0.3 \%$ & 6 & $0.1 \%$ & 0 & $0.0 \%$ & 1,450 & $0.3 \%$ \\
\hline $600,001-700,000$ & 1,042 & $0.2 \%$ & 6 & $0.1 \%$ & 3 & $0.1 \%$ & 1,051 & $0.2 \%$ \\
\hline 700,001 and over & 791 & $0.2 \%$ & 0 & $0.0 \%$ & 0 & $0.0 \%$ & 791 & $0.2 \%$ \\
\hline Total & 433,916 & $100.0 \%$ & 10,103 & $100.0 \%$ & 5,190 & $100.0 \%$ & 449,209 & $1.1 \%$ \\
\hline
\end{tabular}

Table 7. Risk related to payment amount.

\begin{tabular}{|c|c|c|c|c|c|c|c|c|}
\hline \multirow[b]{2}{*}{ Transaction amount } & \multicolumn{2}{|c|}{ Normal account } & \multicolumn{2}{|c|}{ Bad debt account } & \multicolumn{2}{|c|}{ Charge-off account } & \multicolumn{2}{|c|}{ Total account } \\
\hline & No. & Percentage & No. & Percentage & No. & Percentage & No. & Percentage \\
\hline 0 & 426,195 & $98.2 \%$ & 10,066 & $99.6 \%$ & 5,190 & $100.0 \%$ & 441,451 & $98.3 \%$ \\
\hline $1-100,000$ & 6,367 & $1.5 \%$ & 34 & $0.3 \%$ & 0 & $0.0 \%$ & 6,401 & $1.4 \%$ \\
\hline $100,001-200,000$ & 923 & $0.2 \%$ & 3 & $0.0 \%$ & 0 & $0.0 \%$ & 926 & $0.2 \%$ \\
\hline $200,001-300,000$ & 431 & $0.1 \%$ & 2 & $0.0 \%$ & 0 & $0.0 \%$ & 433 & $0.1 \%$ \\
\hline Total & 433,916 & $100.0 \%$ & 10,105 & $100.0 \%$ & 5,190 & $100.0 \%$ & 449,211 & $100.0 \%$ \\
\hline
\end{tabular}

Table 8. Risk related to delinquent flag.

\begin{tabular}{|c|c|c|c|c|c|c|c|c|}
\hline \multirow[b]{2}{*}{ Delinquent flag } & \multicolumn{2}{|c|}{ Normal account } & \multicolumn{2}{|c|}{ Bad debt account } & \multicolumn{2}{|c|}{ Charge-off account } & \multicolumn{2}{|c|}{ Total account } \\
\hline & No. & Percentage & No. & Percentage & No. & Percentage & No. & Percentage \\
\hline 0 & 25,625 & $5.9 \%$ & 215 & $2.1 \%$ & 13 & $0.3 \%$ & 25,853 & $5.8 \%$ \\
\hline 1 & 54,796 & $12.6 \%$ & 340 & $3.4 \%$ & 4 & $0.1 \%$ & 55,140 & $12.3 \%$ \\
\hline 2 & 2,826 & $0.7 \%$ & 689 & $6.8 \%$ & 3 & $0.1 \%$ & 3,518 & $0.8 \%$ \\
\hline 3 & 177 & $0.0 \%$ & 486 & $4.8 \%$ & 4 & $0.1 \%$ & 667 & $0.2 \%$ \\
\hline 4 & 12 & $0.0 \%$ & 425 & $4.2 \%$ & 2 & $0.0 \%$ & 439 & $0.1 \%$ \\
\hline 5 & 8 & $0.0 \%$ & 352 & $3.5 \%$ & 3 & $0.1 \%$ & 363 & $0.1 \%$ \\
\hline 6 & 7 & $0.0 \%$ & 443 & $4.4 \%$ & 27 & $0.5 \%$ & 477 & $0.1 \%$ \\
\hline 7 & 15 & $0.0 \%$ & 230 & $2.3 \%$ & 18 & $0.4 \%$ & 263 & $0.1 \%$ \\
\hline 8 & 1 & $0.0 \%$ & 283 & $2.8 \%$ & 36 & $0.7 \%$ & 320 & $0.1 \%$ \\
\hline 9 & 0 & $0.0 \%$ & 1 & $0.0 \%$ & 3,105 & $59.8 \%$ & 3,106 & $0.7 \%$ \\
\hline B & 84,804 & $19.5 \%$ & 51 & $0.5 \%$ & 8 & $0.2 \%$ & 84,863 & $18.9 \%$ \\
\hline $\mathrm{Z}$ & 265,645 & $61.2 \%$ & 6,590 & $65.2 \%$ & 1,967 & $37.9 \%$ & 274,202 & $61.0 \%$ \\
\hline Total & 433,916 & $100.0 \%$ & 10,105 & $100.0 \%$ & 5,190 & $100.0 \%$ & 449,211 & $100.0 \%$ \\
\hline
\end{tabular}

Table 9. Risk related to B/C. 


\subsection{Fuzzy Input Features}

A fuzzy rule-base system was used to obtain good input features. The fuzzy values were obtained in five steps. First, the membership functions were determined as follows.

$$
\begin{aligned}
& \mu_{A 1}=\left\{\begin{array}{l}
1, x_{1} \leq 20000 \\
\frac{70000-x_{1}}{50000}, 20000<x_{1} \leq 70000 \\
0, x_{1}>70000
\end{array}\right. \\
& \mu_{A 2}=\left\{\begin{array}{l}
0, x_{1} \leq 60000 \\
\frac{x_{1}-60000}{30000}, 60000<x_{1} \leq 900000 \\
\frac{120000-x_{1}}{30000}, 90000<x_{1} \leq 120000 \\
0, x_{1}>120000
\end{array}\right.
\end{aligned}
$$

$$
\mu_{A 3}=\left\{\begin{array}{l}
0, x_{1} \leq 100000 \\
\frac{x_{1}-100000}{50000}, 100000<x_{1} \leq 150000 \\
\frac{200000-x_{1}}{50000}, 150000<x_{1} \leq 200000 \\
0, x_{1}>200000
\end{array}\right.
$$

$$
\mu_{A 4}=\left\{\begin{array}{l}
0, x_{1} \leq 190000 \\
\frac{x_{1}-190000}{110000}, 190000<x_{1} \leq 300000 \\
1, x_{1}>300000
\end{array}\right.
$$

$$
\begin{aligned}
& \mu_{B 1}=\left\{\begin{array}{l}
1, x_{2} \leq 20 \\
\frac{25-x_{2}}{5}, 20<x_{2} \leq 25 \\
0, x_{2}>25
\end{array}\right. \\
& \mu_{B 2}=\left\{\begin{array}{l}
0, x_{2} \leq 24 \\
\frac{x_{2}-24}{15}, 24<x_{2} \leq 39 \\
\frac{54-x_{2}}{15}, 39<x_{2} \leq 54 \\
0, x_{2}>54
\end{array}\right.
\end{aligned}
$$




$$
\begin{aligned}
& \mu_{B 3}=\left\{\begin{array}{l}
0, x_{2} \leq 53 \\
\frac{x_{2}-53}{7}, 53<x_{2} \leq 60 \\
1, x_{2}>60
\end{array}\right. \\
& \mu_{C 1}=\left\{\begin{array}{l}
1, x_{3} \leq 10000 \\
\frac{40000-x_{3}}{30000}, 10000<x_{3} \leq 40000 \\
0, x_{3}>40000
\end{array}\right. \\
& \mu_{C 2}=\left\{\begin{array}{l}
0, x_{3} \leq 30000 \\
\frac{x_{3}-30000}{30000}, 30000<x_{3} \leq 60000 \\
\frac{90000-x_{3}}{30000}, 60000<x_{3} \leq 90000 \\
0, x_{3}>90000
\end{array}\right. \\
& \mu_{C 3}=\left\{\begin{array}{l}
0, x_{3} \leq 80000 \\
\frac{x_{3}-800000}{30000}, 80000<x_{3} \leq 110000 \\
\frac{140000-x_{3}}{30000}, 110000<x_{3} \leq 140000 \\
0, x_{3}>140000
\end{array}\right. \\
& \mu_{C 4}=\left\{\begin{array}{l}
0, x_{3} \leq 130000 \\
\frac{x_{3}-130000}{120000}, 130000<x_{3} \leq 2500000 \\
1, x_{3}>250000
\end{array}\right. \\
& \mu_{D 1}=\left\{\begin{array}{l}
1, x_{4} \leq 10000 \\
\frac{40000-x_{4}}{30000}, 10000<x_{3} \leq 40000 \\
0, x_{4}>40000
\end{array}\right.
\end{aligned}
$$




$$
\mu_{D 2}=\left\{\begin{array}{l}
0, x_{4} \leq 30000 \\
\frac{x_{4}-30000}{30000}, 30000<x_{4} \leq 60000 \\
\frac{90000-x_{4}}{30000}, 60000<x_{4} \leq 90000 \\
0, x_{4}>90000
\end{array}\right.
$$

$$
\mu_{D 3}=\left\{\begin{array}{l}
0, x_{4} \leq 80000 \\
\frac{x_{4}-800000}{30000}, 80000<x_{4} \leq 110000 \\
\frac{140000-x_{4}}{30000}, 110000<x_{4} \leq 140000 \\
0, x_{4}>140000
\end{array}\right.
$$

$$
\mu_{D 4}=\left\{\begin{array}{l}
0, x_{4} \leq 130000 \\
\frac{x_{4}-130000}{120000}, 130000<x_{4} \leq 2500000 \\
1, x_{4}>250000
\end{array}\right.
$$
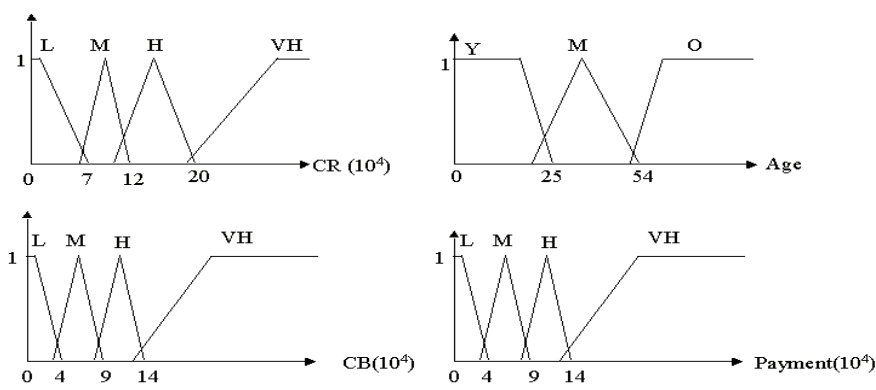

Fig. 1. The respective membership functions for $\mathrm{CR}$ (credit line), age, CB (current balance) and payment.

Hence, $\mu_{A n}, \mu_{B n}, \mu_{C n}$ and $\mu_{D n}$ denote fuzzy membership functions for a credit line, age, current balance and payment, respectively. $n$ is the center of a triangular fuzzy set. The triangular fuzzy sets are plotted in Fig. 1. L, M, H, and VH denote the linguistic variables low, medium, high, very high in the amount feature. $\mathrm{Y}, \mathrm{M}$ and $\mathrm{O}$ denote the linguistic variables young, middle and old for the age feature. Next, the fuzzy rules are created. The rule sets are shown in Tables 10 and 11.

Then, weights are assigned to each linguistic term using subsethood values. Next, the fuzzy membership values are calculated for each linguistic term in each subgroup as given in Tables 10 and 11. The fuzzy membership values are calculated according to each 
classification result. Finally, the classification is calculated using the de-fuzzification to get a single value that represents the output fuzzy set, namely the risk ratio.

\begin{tabular}{ccccc}
\hline \multirow{2}{*}{ Payment } & \multicolumn{4}{c}{ Current balance } \\
\cline { 2 - 5 } & Very high & High & Medium & Low \\
\hline Very high & Common & Good & Excellent & Excellent \\
High & Good & Common & Good & Excellent \\
Medium & Worst & Worst & Common & Good \\
Low & Worst & Worst & Worst & Common \\
\hline
\end{tabular}

Table 10. Current balance to payment linguistic labels matrix.

\begin{tabular}{ccccc}
\hline \multirow{2}{*}{ Age } & \multicolumn{4}{c}{ Credit line } \\
\cline { 2 - 5 } & Very high & High & Medium & Low \\
\hline Young & Good & Common & Common & Worst \\
Middle & Excellent & Good & Common & Common \\
Old & Common & Common & Worst & Worst \\
\hline
\end{tabular}

Table 11. Credit line to age linguistic labels matrix.

\subsection{Input output coding}

Three types of input variables are used, namely qualitative, quantitative (or numeric) and ratio (Durham University, 2008). A binary encoding scheme is used to represent the presence 1, or absence 0, of a particular (qualitative) data. Quantitative data are normalized into the range [0,1]. Ratios are the proportion of related variables calculated to signal the importance of data. We encode ratios by computing the proportion of related variables to describe the importance of the data.

Input variables comprise (1) gender, encoded using one bit ( $0=$ female, $1=$ male), (2) customer age, denotes the customer age between 20 and 80 years, (3) age of the client account, from 1 to 13 years, (4) current balance, denotes the total amount of money owed by cardholders in the range from 1 to 1,000,000, (5) payment amount, denotes the total amount of money debited by cardholders and is in the range from 1 to 1,000,000, (6) transaction amount, denotes the total amount of money consumed by cardholders from 1 to $1,000,000$, (7) delinquent flag, records the status that late payments have been received and is encoded into 3 binary bits, where 000 indicates full pay, 001 minimum monthly payment, 010 delinquent within one month, 011 delinquent within two to four months, 100 delinquent within five to seven months and 101 delinquent above seven months, (8) risk ratio, is given by the FMS and encoded into one ratio bit, (9) payment amount to current balance ratio, denotes solvency and is encoded into one ratio bit.

The two output variables signal the cardholder status. These are coded as 00 normal, 01 bad debt or 10 charge-off accounts.

\section{Experimental Results}

The tools used for implementing the experimental system include JBUILDER 9.0, SQL 2000 and Windows 2000. Input values were normalized to the range from 0 to 1 . After training, 
the neural network is capable of classifying credit status. A predefined threshold of 0.8 was used to detect suspicious cases.

\subsection{Procedure}

A small dataset, provided by a local bank in Taiwan, was used to demonstrate how this method works. This data set contains 449,256 accounts belonging to three classes; namely 433,961 normal accounts, 10,105 bad debt accounts, and 5,190 charge-off accounts. There are only $0.35 \%$ abnormal accounts in practice. The experimental data set is divided into two subsets, namely 3,000 training examples and 10,000 test examples. The training set comprises 1,000 normal accounts, 1,000 bad accounts and 1,000 charge-off accounts. A twoway cross validation table was used to select input features. To obtain good input features a fuzzy rule-based system was incorporated. A risk ratio of variables with fuzzy value was created to enhance the prediction accuracy. After data transformation, the features to be input to the BPN were encoded in the $[0,1]$ interval. The BPN classifies input into one of three classes. The network is repetitively trained with different network parameters until it converges. We randomly selected 3,000 training examples from the total sample, where 1,000 examples were normal accounts, 1,000 were bad dept account and 1,000 were chargeoff accounts.

The neural network learning parameters need to be set to avoid the effect of overfitting and to maintain reasonable performance. Fig. 2 and 3 show system screenshots of the two main views. The learning parameters were tuned by running the simulations multiple times.

The back-propagation network comprised 11 input nodes, 7 hidden nodes, and 2 output nodes. The coding of the output vectors were as follows: bad debt accounts $(1,0)$, charge-off accounts $(0,1)$ and normal accounts $(0,0)$. Table 12 shows BPN typical input output mapping examples.

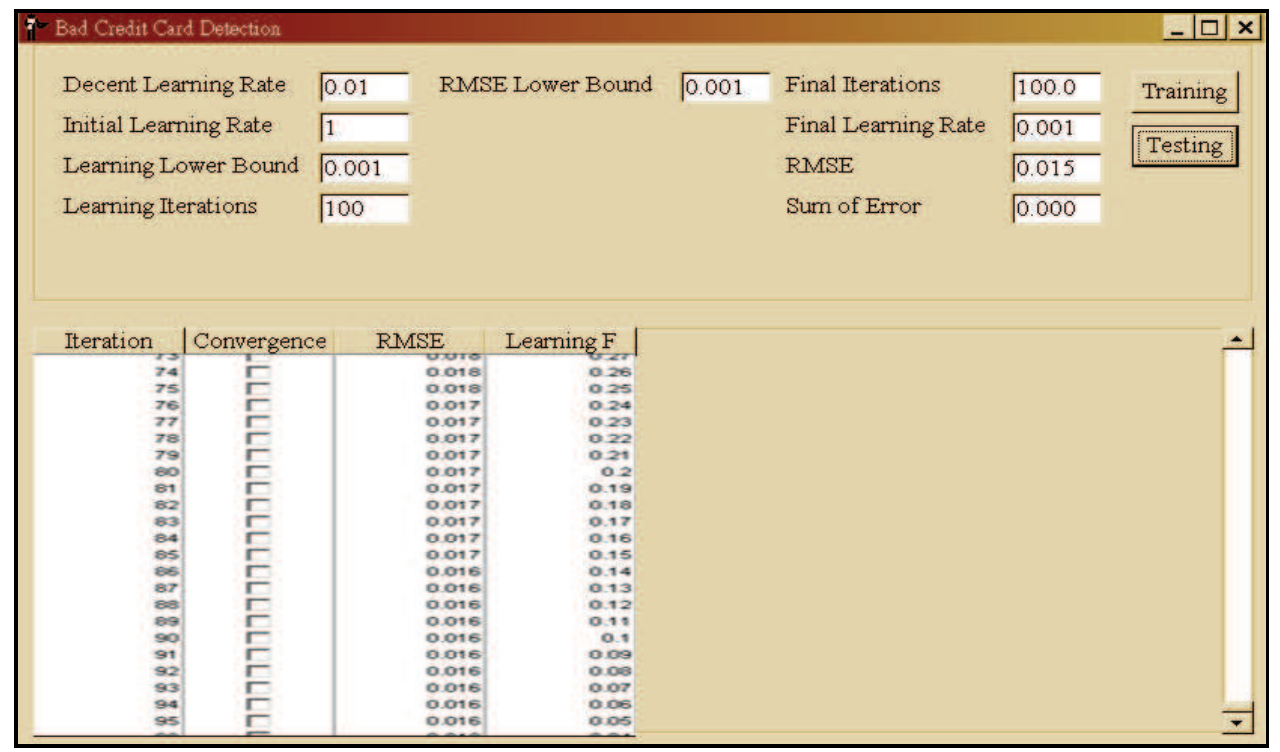

Fig. 2. The training screen. 


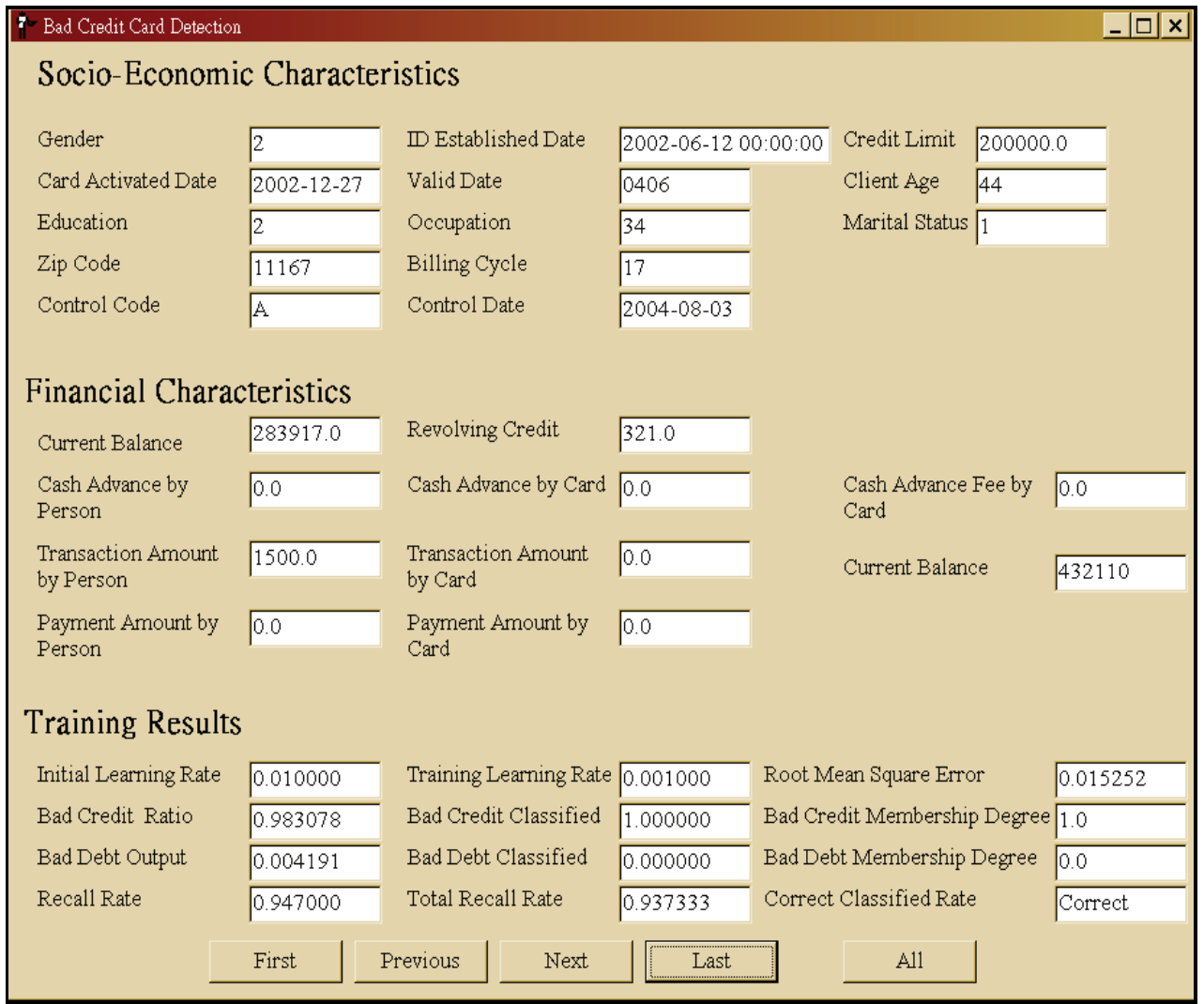

Fig. 3. The test screen.

\begin{tabular}{cccccc}
\hline Name & Type & I/O & bad debt & charge-off & normal \\
\hline X1 & Binary & Input & 1 & 1 & 0 \\
X2 & Quantification & Input & 0.25 & 0.31 & 0.51 \\
X3 & Quantification & Input & 0.18 & 0.23 & 0.23 \\
X4 & Quantification & Input & 0.45 & 0.95 & 0.17 \\
X5 & Quantification & Input & 0.18 & 0.15 & 0.36 \\
X6 & Quantification & Input & 0.15 & 0.15 & 0.16 \\
X7 & Binary & Input & 010 & 101 & 000 \\
X8 & Ratio & Input & 0.44 & 0.95 & 0.16 \\
X9 & Ratio & Input & 0.29 & 0.15 & 0.15 \\
\hline O1 & Binary & Output & 0.9997 & 0.0091 & 0.0215 \\
O2 & Binary & Output & 0.0002 & 0.9905 & 0.0286 \\
\hline
\end{tabular}

Table 12. Neural network mapping examples. 


\subsection{Performance Evaluation}

Performance was measured in terms of precision and recall. Precision is defined as the proportion of classified instances that were correctly classified, and recall as the proportion of instances classified correctly (Cohn, 2003), or formally

$$
\begin{gathered}
\text { recall }=\frac{|A(q) \cap R(q)|}{|R(q)|}, \\
\text { precision }=\frac{|A(q) \cap R(q)|}{|A(q)|} .
\end{gathered}
$$

\subsection{Detection Performance}

We started with a network with all possible input nodes. But all possible nodes are never needed to represent a system. Therefore, we used two-way cross validations to filter off redundant input features. Besides, we designed risk ratio of variables to raise the performance. We fixed the network parameters and set the initial learning rate to 10 with a decreasing rate of 0.001 . We consolidated the performance achieved with the fuzzy BPN and the non-fuzzy BPN (see Table 13). Clearly, the fuzzy BPN yields better results than the conventional BPN.

\begin{tabular}{cccccc}
\hline \multirow{2}{*}{ Iterations } & \multicolumn{2}{c}{ Proposed detection model } & & \multicolumn{2}{c}{ Conventional BPN model } \\
\cline { 2 - 3 } \cline { 5 - 6 } & Recall (R1) & Precision (P1) & & Recall (R2) & Precision (P2) \\
\hline 100 & $85.20 \%$ & $79.00 \%$ & & $75.80 \%$ & $65.00 \%$ \\
200 & $87.95 \%$ & $84.00 \%$ & & $80.15 \%$ & $72.00 \%$ \\
500 & $88.30 \%$ & $83.90 \%$ & & $81.30 \%$ & $73.00 \%$ \\
1000 & $90.00 \%$ & $85.00 \%$ & & $83.60 \%$ & $76.80 \%$ \\
2000 & $95.50 \%$ & $95.00 \%$ & & $86.00 \%$ & $80.00 \%$ \\
Average & $95.35 \%$ & $94.97 \%$ & & $86.15 \%$ & $80.20 \%$ \\
\hline
\end{tabular}

Table 13. The performance of the proposed detection model versus conventional BPN detection.

Additionally, a dataset comprising 10,000 simulated entries were used to evaluate and validate the system, where different normal to abnormal data ratios were considered to diagnose different behaviours. The results are listed in Table 14, and each experiment shows the size of the detected set, the number of addressed problems, the total precision $\mathrm{P}$ and the 
total recall $\mathrm{R}$ rates. This experimental evidence demonstrates that the strategy is capable of effectively tackling more than $90 \%$ of the problems.

\begin{tabular}{ccccccc}
\hline \multirow{2}{*}{ Result } & \multirow{2}{*}{ Size } & \multicolumn{2}{c}{ Number of addressed problems } & \multirow{2}{*}{ Recall } & \multirow{2}{*}{ Precision } \\
\cline { 3 - 5 } & & Normal & Bad debt & Charge-off & & \\
\hline Test Data-1 & 282 & 9706 & 137 & 145 & $94.00 \%$ & $95.59 \%$ \\
Test Data-2 & 4512 & 5177 & 2036 & 2476 & $90.24 \%$ & $93.55 \%$ \\
Test Data-3 & 5372 & 4068 & 2420 & 2952 & $89.53 \%$ & $90.56 \%$ \\
\hline
\end{tabular}

Table 14. The comparisons of test results from different iterations.

\subsection{Implications}

The strategy outlined herein could be used for risk management, analysis of business rules, delinquent diagnosis and abnormal accounts forecasting. Risk management helps reduce issuers' losses due to bad debt. When detecting bad debt accounts, the issuers can reduce the credit line. Moreover cardholders can be offered realistic loan plans to help them overcome their financial difficulties (Lin, 2003). Analysis of business rules is used to establish a way to normalize the analysis that facilitates to compare the business rules across various types of credit card accounts. We could take these rules to develop a business model in the knowledge management system. Delinquent diagnosis is used to analyze the existing delinquent factors as a high-level understanding of process and control systems of an application. Delinquent diagnosis is a way to monitor the delinquent accounts early to avoid losses due to bad debt. Abnormal account forecasting is commonly used to recognize portfolio dynamics and behaviour patterns.

\section{Conclusions and Future Work}

A novel scheme for the bad credit account detection was proposed. A fuzzy rule-based system was used to provide inputs for a back-propagation neural network that was used for classifying accounts. The proposed system has been tested on real credit data and it is capable of detecting bad accounts in the large data set with a success rate of more than $90 \%$. Future work includes integrating the proposed system with credit card risk management systems and the introduction of noise reduction mechanism for discarding outlier accounts, i.e., observations that deviates so much from other observations as to arouse suspicion that it was generated by a different mechanism. Finally, it would be desirable to integrate other AI algorithms (e.g., GA) with data mining to enhance predictive accuracy and apply the algorithm to relational (e.g., spatial) data.

\section{References}

Aleskerov, E.; Freisleben, B. \& Rao, B. (1997). CARDWATCH: a neural network based database mining system for credit card fraud detection. Proceedings of IEEE Int. Conf. on Computational Intelligence for Financial Engineering, pp. 220-226, NY, USA, March 1997. 
Brause, R.; Langsdorf, T. \& Hepp, M. (1999). Neural data mining for credit card fraud detection. Proceedings of 11th IEEE Int. Conf. on Tools with Artificial Intelligence, pp. 103-106, Chicago, IL, USA, November 1999.

Burns, P. \& Stanley, A. (2001). Managing consumer credit risk. Federal Reserve Bank of Philadelphia Payment Cards Center Discussion Paper, no. 01-03, pp. 1-7, November 2001.

Chakraborty, D. \& Pal, N.R. (2001). Integrated feature analysis and fuzzy rule-base system identification in a neuro-fuzzy paradigm. IEEE Trans. Systems, Man, and Cybernetics, vol. 31, no. 4, pp. 391-400, June 2001.

Chiang, S.C. (2003). The relationship between credit-evaluation factors and credit card default riskan example of the credit cards issued by the a financial institute in Taiwan. Master Thesis, Department of Insurance, Feng Chia University, Taichung, Taiwan, June 2003.

Cohn, T. (2003). Performance metrics for word sense disambiguation. Proceedings of the Australasian Language Technology Workshop, pp. 49-56, Victoria, Australia, December 2003.

Huang, Y.-P.; Chang, T.-W.; Chen, Y.-R. \& Sandnes, F.E. (2008). A back propagation based real-time license plate recognition system. Int. Journal of Pattern Recognition and Artificial Intelligence, vol. 22, no. 2, pp. 233-251, March 2008.

Huang, Y.-P. \& Hsieh, W.-J. (2003). The application of grey model and back-propagation network to establish the alarm mechanism for the premium rate service. Journal of Grey System, vol. 6, no. 2, pp. 75-88, December 2003.

Huang, Y.-P.; Hsu, L.-W. \& Sandnes, F.E. (2007). An intelligent subtitle detection model for locating television commercials. IEEE Trans. on Systems, Man, and Cybernetics, Part B: Cybernetics, vol. 37, no. 2, pp. 485-492, April 2007.

Huang, Y.-P.; Huang, Y.-H. \& Sandnes, F.E. (2006). A fuzzy inference model-based nonreassuring fetal status monitoring system. Int. Journal of Fuzzy Systems, vol. 8, no. 1, pp. 57-64, March 2006.

Kijsirikul, B. \& Chongkasemwongse, K. (2001). Decision tree pruning using backpropagation neural networks. Proceedings of IEEE Int. Conf. on Neural Networks, vol. 3, pp. 18761880, Washington D.C., USA, Month 2001.

Kuo, Y.F.; Lu, C.T.; Sirwongwattana, S. \& Huang, Y.-P. (2004). Survey of fraud detection techniques. Proceedings of IEEE Int. Conf. on Networking, Sensing and Control, pp. 749754, Taipei, Taiwan, March 2004.

Lee, H.M.; Chen, C.M.; Chen, J.M. \& Jou, Y.L. (2001). An efficient fuzzy classifier with feature selection based on fuzzy entropy. IEEE Trans. on Systems, Man and Cybernetics, vol. 31, no. 3, pp.426-432, June 2001.

Lee, M.H. (2002). A study on the credit risk of the credit card holder. Master Thesis, Department of Insurance, Feng Chia University, Taichung, Taiwan, June 2001.

Lin, C.J. (2003). Data mining for risk improving of credit card. Master Thesis, Department of Computer Science and Engineering, Tamkang University, Taipei, Taiwan, June 2003.

Lin, J. (2005). Interest-rate cap dropped as bankers offer relief plan. Taipei Times, Friday, Dec. 16, 2005.

$\mathrm{Yu}, \mathrm{H} . \mathrm{H}$. (2003). The research of applying improved artificial neural network to credit card customer relationship management. Master Thesis, Department of Business Management, National Taipei University of Technology, Taipei, Taiwan, June 2003. 
Zhang, D. \& Zhou, L. (2004). Discovering golden nuggets: data mining in financial application. IEEE Trans. on Systems, Man, and Cybernetics-Part C: Applications and Reviews, vol. 34, no. 4, pp. 513-522, November 2004.

Cardservice International website (2008), http:/ / www.aboutcsi.com.

Central Bank of China website (2008), http://www.cbc.gov.tw.

Durham University website (2008), http:/ / www.dur.ac.uk.

Indiana University Office of the Treasurer website (2008), http://www.indiana.edu.

National Credit Card Center of R.O.C website (2008), http://www.nccc.com.tw.

ShopSite website (2008), http:/ / www.shopsite.com.

Taiwan Financial Supervisory Commission website (2008), http://www.fscey.gov.tw.

Taiwan Joint Credit Information Center website (2008), http://www.jcic.org.tw.

The International Commercial Bank of China website (2008), http:/ / www.icbc.com.tw. 


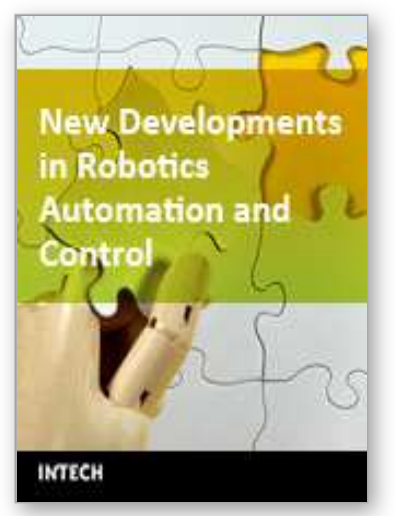

\section{New Developments in Robotics Automation and Control \\ Edited by Aleksandar Lazinica}

ISBN 978-953-7619-20-6

Hard cover, 450 pages

Publisher InTech

Published online 01, October, 2008

Published in print edition October, 2008

This book represents the contributions of the top researchers in the field of robotics, automation and control and will serve as a valuable tool for professionals in these interdisciplinary fields. It consists of 25 chapter that introduce both basic research and advanced developments covering the topics such as kinematics, dynamic analysis, accuracy, optimization design, modelling, simulation and control. Without a doubt, the book covers a great deal of recent research, and as such it works as a valuable source for researchers interested in the involved subjects.

\section{How to reference}

In order to correctly reference this scholarly work, feel free to copy and paste the following:

Yo-Ping Huang, Frode Eika Sandnes, Tsun-Wei Chang and Chun-Chieh Lu (2008). Intelligent Detection of Bad Credit Card Accounts, New Developments in Robotics Automation and Control, Aleksandar Lazinica (Ed.), ISBN: 978-953-7619-20-6, InTech, Available from:

http://www.intechopen.com/books/new_developments_in_robotics_automation_and_control/intelligent_detecti on_of_bad_credit_card_accounts

\section{INTECH}

open science | open minds

\section{InTech Europe}

University Campus STeP Ri

Slavka Krautzeka 83/A

51000 Rijeka, Croatia

Phone: +385 (51) 770447

Fax: +385 (51) 686166

www.intechopen.com

\section{InTech China}

Unit 405, Office Block, Hotel Equatorial Shanghai

No.65, Yan An Road (West), Shanghai, 200040, China

中国上海市延安西路65号上海国际贵都大饭店办公楼 405 单元

Phone: +86-21-62489820

Fax: $+86-21-62489821$ 
(C) 2008 The Author(s). Licensee IntechOpen. This chapter is distributed under the terms of the Creative Commons Attribution-NonCommercialShareAlike-3.0 License, which permits use, distribution and reproduction for non-commercial purposes, provided the original is properly cited and derivative works building on this content are distributed under the same license. 